\title{
Lattice-Boltzmann Based Large-Eddy Simulations Applied to Industrial Flows
}

\author{
Jos Derksen \\ Kramers Laboratorium, Applied Physics Department, Delft University of Technology, Prins \\ Bernhardlaan6, 2628 BW Delft, The Netherlands \\ jos@klft.tn.tudelft.nl
}

\begin{abstract}
A procedure for simulating single-phase, turbulent fluid flow based on the lattice-Boltzmann method is described. In the large-eddy approach to turbulence modeling, the Smagorinsky subgrid-scale model, supplemented with wall damping, is applied exclusively. The assumptions underlying the model are discussed. Simulation results of some sample flow systems from chemical engineering practice are presented, with an emphasis on experimental validation, and grid effects.
\end{abstract}

\section{Introduction}

The performance of many industrial processes relies on turbulent flows. In agitated tanks, in tubular reactors, in bubble columns, and in fluidized beds, the turbulent fluctuations (that cover broad spatial and temporal spectra) enhance heat and mass transfer, control bubble and particles size distributions due to break-up and coalescence, and create contact area to facilitate chemical reactions. As a result, there definitely is a need for good insight, and accurate predictions of turbulent flows in process equipment. Computational Fluid Dynamics (CFD) is one of the tools that (to some extent) might be able to satisfy this need. In CFD, the mass and momentum balances that govern fluid flow are solved numerically. Because one of the characteristics of turbulence is its wide range of scales, a full representation (i.e. a Direct Numerical Simulation (DNS)) of the flow would require unrealistic amounts of computer resources. As an example, consider the flow with a macroscopic Reynolds number $R e=\frac{U L}{v}=10^{4}$. The smallest dynamic flow scale $\eta_{K}$ (i.e. the Kolmogorov scale) is related to the macro-scale through $\eta_{K}=L R e^{-3 / 4}$. A DNS would require a spatial resolution at least equal to $\eta_{K}$. As a result, to cover all length scales of the flow in three dimensions, a grid of $1,000^{3}=10^{9}$ cells has to be defined. Nowadays, these numbers start becoming feasible on massively parallel computer platforms.

There are, however, a few reasons not to pursue DNS of strongly turbulent flows in chemical engineering. In the first place, with a view to industrial applications, $R e=10^{4}$ is still considered a low value. In full-scale process equipment, the Reynolds number easily amounts to several millions. In the second place, the purpose of our research is 
to apply CFD as a scale-up, or design tool. This implies that it has to be possible to study alternative reactor designs, and process conditions within a limited amount of time, and on computer platforms widely available in academia and industry. In the third place, again with a view to the applications, the flow simulations are not the final goal of our efforts. They need to be related to the process, which next to fluid dynamics involves other physical and chemical phenomena. A good balance has to be found with respect to the computational efforts for modeling the various aspects of the process.

The alternative to DNS is turbulence modeling. Based on assumptions regarding turbulent fluctuations on the small scales (e.g. related to their supposedly universal character, their tendency to isotropy and equilibrium) turbulence models can be devised that distinguish between resolved and unresolved (i.e. modeled) phenomena. In the field of turbulence modeling, a division can be made between models based on the Reynolds Averaged Navier-Stokes (RANS) equations, and Large-Eddy Simulations (LES). For industrial (chemical engineering) applications, RANS modeling has a much longer tradition than LES. This is related to the lesser demand for computational resources of the former approach. In a RANS simulation, the grids can be coarser since only the average flow is explicitly simulated, and (in most cases) timedependence is not taken into account. Furthermore, use can be made of symmetry in the boundary conditions. A large-eddy simulation shows a strong resemblance to a direct simulation: the three-dimensional, time-dependent Navier-Stokes equations are discretized to a grid that is (necessarily) too coarse. By viewing the flow field on the grid as a low-pass filtered representation of the true field, and by modeling the interaction between the filtered flow and the (unresolved) flow at length scales smaller than the grid spacing (the subgrid-scale (SGS) motion), the flow system is simulated.

The three-dimensionality, and time-dependence of LES make them much more computationally demanding than RANS simulations. Fine grids are required to meet the criteria for SGS modeling in LES [1]. I think, however, that it is worthwhile pursuing an LES approach to industrial flows for the following reasons. In the first place, by resolving the time-dependent flow field at high resolution, we are better able to account for the non-linearity's encountered in process modeling (e.g. chemical reactions, nucleation of crystals in flowing supersaturated liquids, transport of solid particles in a gas stream). In the second place, better flow field predictions are expected from LES in the case of inherently transient flows. By its very nature, turbulence is time-dependent in an incoherent and unpredictable way. If, on top of the turbulent fluctuations, coherent time-dependence is introduced through boundary conditions (as is the case in turbo machinery applications, or in stirred tanks), or through a macroscopic instability in the flow (such as vortex core precession in swirling flow), RANS based models might fail. In the closure models for the RANS equations, no clear spectral distinction exists between resolved and unresolved fluctuations (this in contrast to LES, where the grid makes the distinction). As a result, RANS closure models that are designed for non-coherent, turbulent fluctuations might (erroneously) affect the macroscopic, coherent fluctuations brought about by e.g. the boundary conditions.

In this paper, some examples of LES applied to industrially relevant, turbulent flows will be discussed. But first, our numerical method will be treated, which is based on the lattice-Boltzmann method. Second, a brief account of subgrid-scale mod- 
eling will be given. The examples comprise of agitated flow systems (stirred tanks), and swirling flows (with cyclone separators as the application).

\section{Lattice-Boltzmann Method}

Lattice-Boltzmann modeling of fluid flow has evolved from lattice-gas cellular automata (see e.g. the text book by Rothman \& Zaleski [2]). The starting point of these particle-based methods is principally different from that of more traditional ways of numerical fluid flow modeling, such as finite element or finite volume methods. In the latter method, the continuous equations governing fluid flow are discretized. Latticegas and lattice-Boltzmann schemes start with a discrete system that can be proven to obey the Navier-Stokes equations. The discrete system that mimics fluid flow is a set of (fictitious) particles residing on a uniform lattice. Every time step, particles move to neighboring lattice sites, where they exchange momentum (i.e. collide) with particles coming in from other directions. The collision rules, and the topology of the lattice can be defined in such a way that macroscopically the Navier-Stokes equations are recovered [3]. The elegance of the method is its simplicity, and the locality of its operations. This locality (i.e. the absence of global numerical operations) is responsible for the high parallel efficiency of the method.

Lattice-Boltzmann methods have been applied to many problems involving fluid flow (see e.g. the review article by Chen \& Doolen [3]). Many of the applications involve laminar flow in complexly shaped geometries (e.g. porous media modeling), interfacial phenomena, and multiphase flows. Large-eddy simulations of turbulent flows with lattice-Boltzmann schemes have been reported by Hou et al. [4], Somers [5], and Eggels [6].

We use PC based computer hardware for our lattice-Boltzmann based large-eddy simulations. By connecting the PC's (Dual-Pentium III $700 \mathrm{MHz}$ machines running under Linux) through $100 \mathrm{BaseTX}(100 \mathrm{Mbit} / \mathrm{s})$ switched Ethernet, a Beowulf cluster is formed that can efficiently handle large jobs. A lattice-Boltzmann simulation on $360^{3}$ (i.e. 47 million) cells, typically requires ten processors, 4 Gbyte of memory, and some 40 hours of wall-clock time to simulate one integral time scale (e.g. one impeller revolution in a stirred tank) of the flow.

\section{Subgrid-scale Modeling}

The most widely used model in LES is the Smagorinsky model [7]. It is an eddyviscosity SGS model based on the assumption of local equilibrium between production and dissipation of turbulent kinetic energy. The expression for the eddy-viscosity $\left(v_{e}\right)$ reads:

$$
v_{e}=\lambda_{\text {mix }}^{2} \sqrt{\frac{1}{2}\left(\frac{\partial u_{i}}{\partial x_{j}}+\frac{\partial u_{j}}{\partial x_{i}}\right)^{2}}
$$


with $\lambda_{\text {mix }}$ a mixing length, and $u_{i}$ the resolved (i.e. the grid scale) velocity in the $i$-th coordinate direction. In the Smagorinsky model, the mixing length is proportional to the grid spacing $\Delta: \lambda_{\text {mix }}=c_{s} \Delta$, with $c_{s}$ a constant. This constant can be estimated from turbulence theory under the assumption of local equilibrium: $c_{s}=0.165$. Note that the eddy-viscosity depends on the grid spacing. In case the grid spacing approaches zero, the eddy-viscosity vanishes, which should be, as zero grid spacing implies a direct simulation (i.e. a simulation without SGS model). In many simulations, especially of shear driven turbulence, the value of $c_{s}$ is chosen between 0.06 and 1.2 [8]. This is because, especially near walls, the equilibrium assumption breaks down, and the length scales become too small to resolve. By decreasing the mixing length relative to the grid, the lack of spatial resolution is (artificially) compensated for.

The vicinity of walls poses more problems to the Smagorinsky model: the turbulence near the wall becomes anisotropic (fluctuations in the wall-normal direction are suppressed), and at the wall the SGS Reynolds stresses should become zero. These effects are not a priori accounted for in the model. The eddy-viscosity concept implies isotropic turbulence at the subgrid-scales throughout the flow domain, including the wall region. The problem of non-zero wall stresses can be repaired by applying walldamping functions that force the eddy-viscosity (and as a result the SGS Reynolds stresses) to zero. In our studies we apply Van Driest wall damping functions [9].

In summary, the Smagorinsky model is not an obvious choice when attempting LES of flows in complexly shaped domains with intrinsic time-dependence. In the first place because of the presence of walls that pose principle problems related to anisotropy and resolution. In the second place because of the time-dependence, that will keep the flow off-equilibrium at least in parts of the flow domain. Dynamic SGS modeling (as introduced by Germano et al. [10]) might overcome some of the difficulties

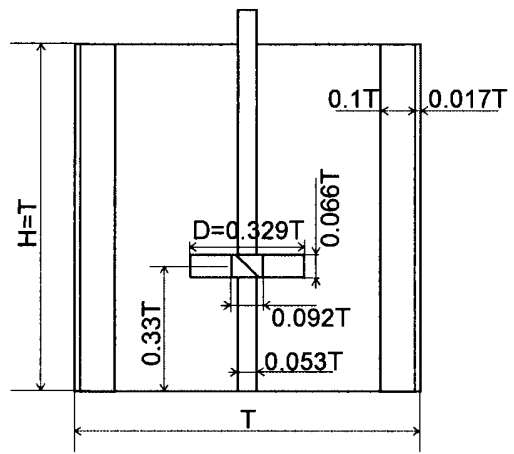

Figure 1. Left: stirred tank geometry. The tank has four, $90^{\circ}$ spaced baffles, with a small wall clearance. The impeller has four pitched blades under a $45^{\circ}$ angle. The top surface is closed. Right: single realization of the flow in a vertical plane midway between two baffles in terms of velocity vectors at $R e=7,300$. The simulation employed a $240^{3}$ grid. The spatial resolution of the vector plot is (in axial and radial direction) twice as low as the actual resolution of the simulation.

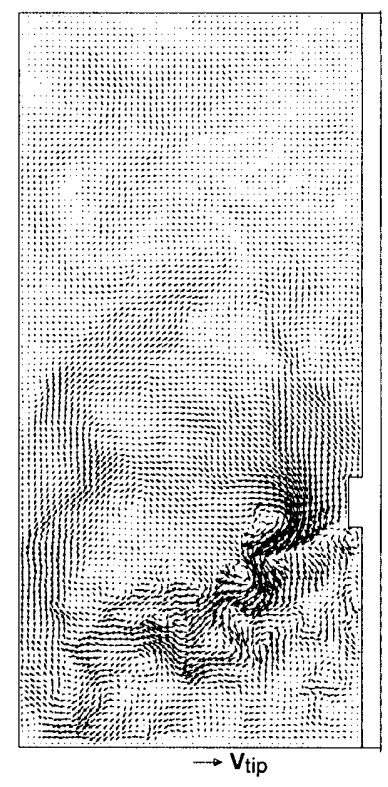


discussed here. In dynamic modeling, a double low-pass filter operation is applied to the flow, with the aim of determining local values of the "constant" $c_{s}$. However, some problems (e.g. related to the stability of the method), even in simple flow geometries have been reported [1].

For the lack of better models, the Smagorinsky SGS model has been applied exclusively in our studies. The issues raised above with respect to the validity of this SGS model require critical evaluation and experimental validation of the simulated flow fields. In this respect, the extent to which the solution depends on the grid spacing requires special attention.

\section{Agitated Flows}

Blending, crystallization, suspension polymerization, particle coating, are some examples of processes carried out in stirred tanks. A revolving impeller generates a turbulent flow in a cylindrical vessel that (apart from the impeller) contains all sorts of static internals, e.g. baffles to prevent solid body rotation of the fluid, draft tubes to direct the flow, heat exchangers, nozzles for injection of reactants, etc. From experiments (e.g. [11]) it is well known that impellers generate complex vortex structures. These vortex structures are largely responsible for the mixing capacity of the flow system, and are associated with strong, anisotropic turbulent activity.

In order to standardize experimental and numerical work on stirred tank flow, a few de facto standard tank and impeller configurations can be identified. A standard tank has a height to diameter ratio of one, and has four, $90^{\circ}$-spaced baffles at its perimeter with a width of one tenth of the tank diameter. In a previous paper, large-eddy simulations on the flow driven by a Rushton turbine have been discussed [12]. In the present paper, results on flow simulations in a standard tank, equipped with a $45^{\circ}$ pitched blade turbine will be presented. The geometry is fully defined in Fig. 1. This

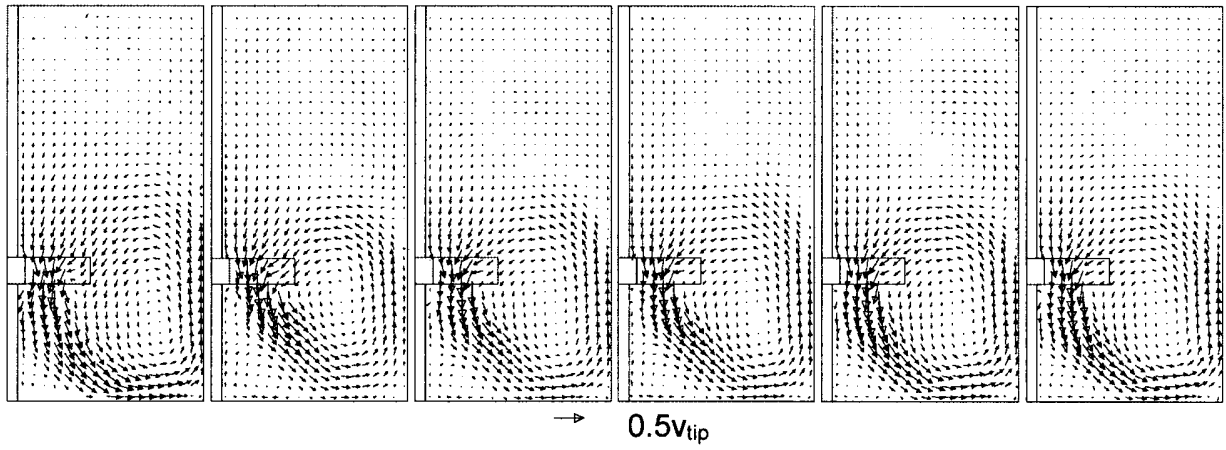

Figure 2. Phase-averaged flow in the vertical plane midway between two baffles at $R e=7,300$. From left to right: experimental data [11]; LES on a $120^{3}$ grid without wall damping functions; LES on a $240^{3}$ grid without wall damping functions; LES on a $240^{3}$ grid with wall damping functions; LES on a $360^{3}$ grid without wall damping functions; LES on a $360^{3}$ grid with wall damping functions. Per simulation, the averaging extended over at least 12 impeller revolutions. The LES results have been linearly interpolated to the experimental grid. 
geometry was chosen because of the availability of a detailed experimental study by Schäfer et al. [11] on the flow field inside such a tank at $R e=\frac{N D^{2}}{v}=7,300$ (with $N$ the impeller's angular velocity in rev./s, $D$ the tank diameter, and $v$ the kinematic viscosity of the fluid).

In Fig. 1, a snapshot of the simulated flow field is presented. From this figure, the inhomogeneous character of the flow is obvious. A strongly turbulent, inclined impeller stream, directs fluid from the impeller towards the tank bottom. At the tank's outer wall, fluid (on average) moves in the upward direction. The stream at the tank wall is not strong enough to reach the top surface of the tank. About halfway the tank height, fluid near the outer wall is sucked into the impeller region. As a result, there is a large part of the tank volume with quiescent fluid.

In Fig. 2, experiment and simulations are compared in terms of the phase-average flow (i.e. the flow averaged over all angular positions of the impeller), in the vertical plane located midway between two baffles. The major flow features in the experimental field are the large, counter-clockwise circulation loop that approximately covers the lower half of the tank, and a clockwise rotating secondary loop in the vicinity of the bottom and the axis. The top half of the tank hardly shows coherent flow structures. In the simulations, presented in Fig. 2, the grid size has been varied, and wall damping has been switched off and on. The coarsest grid $\left(120^{3}\right.$ nodes $)$ has trouble in properly representing the primary circulation (its core being too high, and at too small radial position), as well as the secondary circulation (it extends almost to the impeller, whereas in the experiments it is limited to approximately $35 \%$ of the impeller bottom clearance). Predictions on the primary circulation significantly improve in case of the $240^{3}$ grid. The influence of the wall damping functions is limited, and mainly related to the separation point of the primary circulation from the tank's outer wall: wall damping slightly shifts the separation point upwards, in better agreement with experiments. The secondary circulation is still too big on the $240^{3}$ grid, its vertical size is some $50 \%$ of the impeller clearance, irrespective of wall damping. The impact of wall
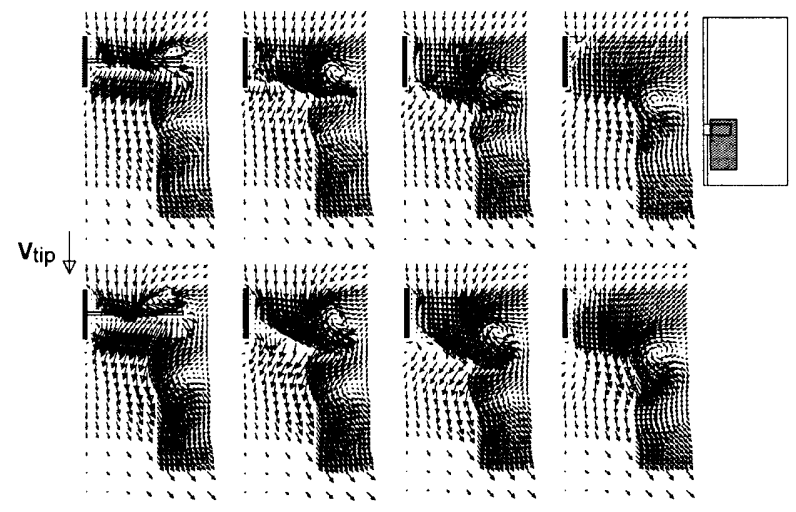

Figure 3. Phase-resolved velocity field at four different impeller angles (from left to right: $0^{\circ}$; $16^{\circ} ; 30^{\circ} ; 60^{\circ}$ ) in the vicinity of the turbine (the field of view is indicated in the right diagram) at $R e=7,300$. Top row: experiments [11]. Bottom row: LES on a $240^{3}$ grid. The LES results have been linearly interpolated to the experimental grid. 
damping functions on the simulation results is most pronounced for the $360^{3}$ grid. This indicates that the grid has become sufficiently fine to feel the influence of details of the boundary layer. The separation point is located much too low for the $360^{3}$ simulation without wall damping, but is almost perfectly predicted with wall damping. The size of the secondary circulation strongly benefits from the finer grid: it corresponds well with the experimental data.

Phase-averaged data obscure the details of the flow around the impeller blades. By conditionally averaging the flow at specific impeller positions, the action of the impeller on the fluid can be studied in more detail. In Fig. 3, phase-resolved LES results are confronted with experimental data [11]. Most striking flow feature is the tip vortex that is generated at the trailing edge of the inclined blade. It is a persistent phenomenon, in the sense that after two blade passages the vortex can still be distinguished in the phase-resolved flow field. The position, size, and shape (squeezed for small angular impeller positions, circular for larger angles) are well represented by the LES.

\section{Swirling Flows}

Swirling flows are challenging from a CFD viewpoint. They exhibit all sorts of, often counterintuitive, flow phenomena. A few examples are flow reversal, strongly anistropic turbulence, subcritical behavior, and (quasi) periodic fluctuations [13]. Our motivation for swirling flow studies lies in their application for separation of process streams containing phases with different density: Hydrocyclones are widely employed in the oil industries for separation of water and oil; gas cyclones are used for separating solid particles from a gas stream. Results on our large-eddy simulations of cyclonic flow have been published elsewhere [14]. In the present paper, I would like to focus on a different flow geometry, see Fig. 4. This so-called vortex tube was used in an experimental study by Escudier et al. [15]. In the latter article, it was demonstrated that downstream geometrical conditions had strong impact on the swirling flow in the entire geometry. The high-quality LDA data, and the visualizations presented in [15] were used to critically test the performance of our simulation procedures. One of the interesting issues here is to what extent the (isotropic) Smagorinsky SGS model [7] is capable of representing a flow system from which it is well know that isotropic RANS models (e.g. the $k$ - $\varepsilon$ model) are inadequate [16].

In the experimental paper, the exit diameter was varied between $D_{e}=D$ to $D_{e}=0.18 D$, with $D$ and $D_{e}$ defined in Fig. 4 . For the LES, three geometries were selected: $D_{e}=0.73 D, D_{e}=0.45 D$, and $D_{e}=0.33 D$. For these three cases, the Reynolds
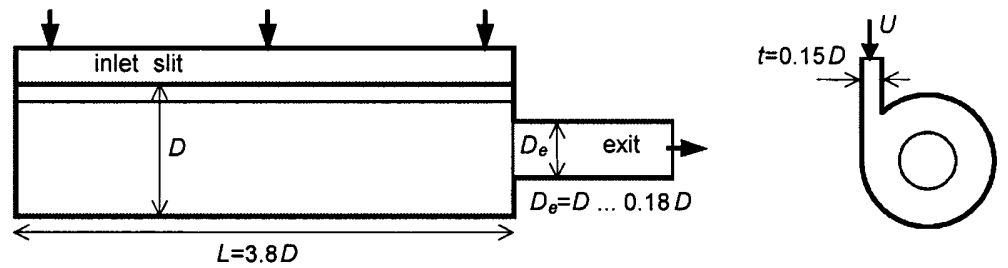

Figure 4. Vortex tube geometry as defined by Escudier et al. [15]. Fluid enters through a tangential slit, and exits through a contraction. 
number was fixed at $R e=\frac{U t}{v}=1,900$, with $U$ the (superficial) inflow velocity, and $t$ the width of the inlet slit (see Fig. 4). This (atypical) definition of Re follows the experimental work. Reynolds numbers e.g. based on the superficial exit velocity, and diameter of the exit tube show much higher values (up to 51,000 for $D_{e}=0.33 D$ ), indicating that subgrid-scale modeling is definitely necessary. The three geometrical layouts chosen were discretized on two grids: a coarse grid with $D=82 \Delta$, and a fine grid with $D=132 \Delta$, as a result, six simulation cases were defined. From our experience with cyclonic flow [14], we know that wall damping functions have to be applied to get a realistic representation of the swirling flow.

Velocity profiles of the tangential and axial velocity are presented in Fig. 5. The axial (i.e. $z$ ) position of the profiles, as indicated in the captions, is measured from the closed end wall of the vortex tube (located at the left side in Fig. 4). The profiles at $z=0.7 D$ are close to the end wall, the profiles at $z=3.6 D$ are close to the contraction into the exit tube. The impact of the exit pipe diameter on the entire flow is evident. The levels of the axial and tangential velocities strongly increase if the exit pipe diameter is made smaller (please note the different vertical scales in Fig. 5). Qualitatively, the flow features apparent from the profiles are well represented by the LES: the Rankine type of vortices as indicated by the tangential velocity profiles; the axial velocity deficit near the vortex core for the larger exit pipe configurations; the pres-
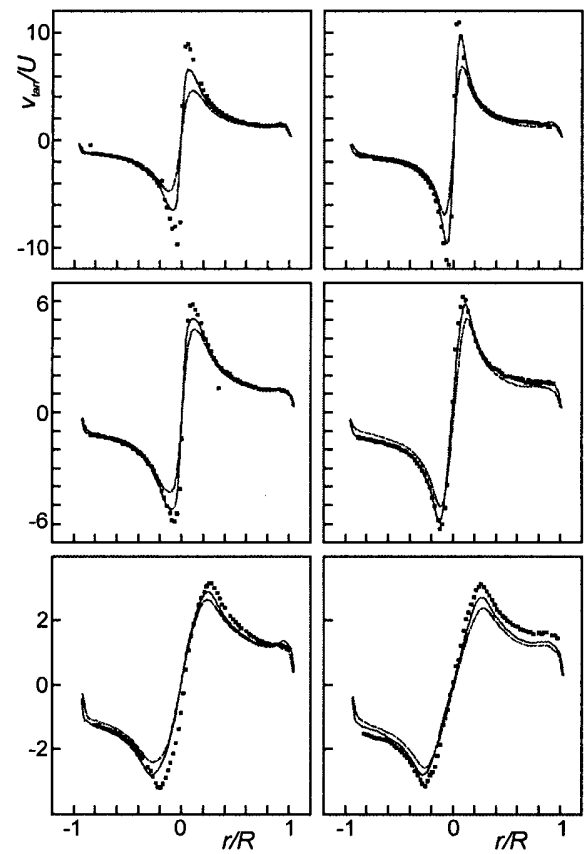
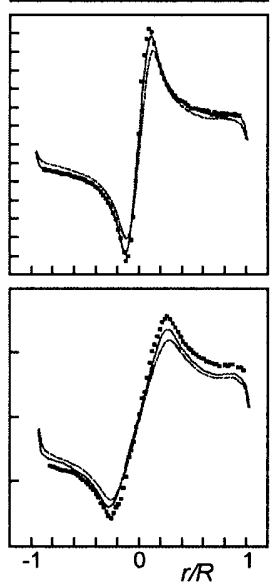
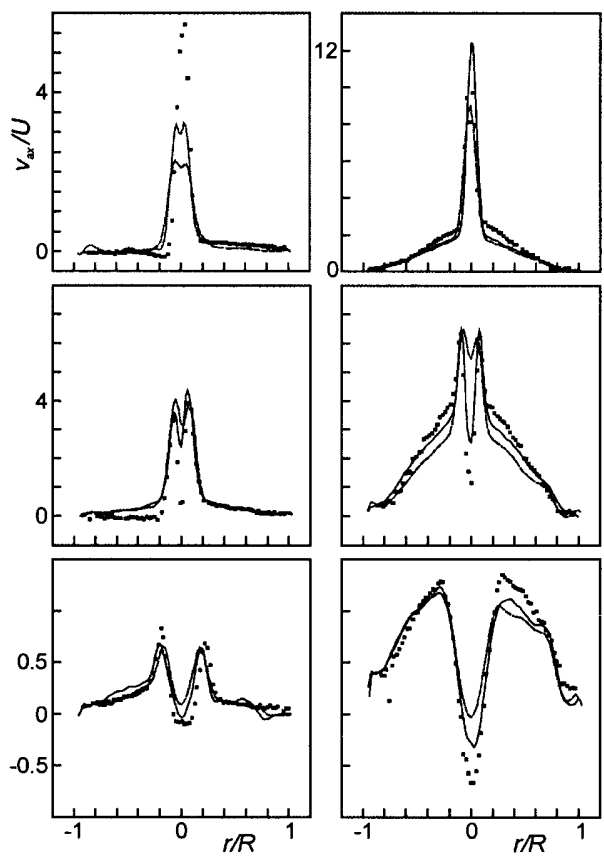

Figure 5. Average tangential velocity (left two columns), and axial velocity (right two columns). Comparison between experiments (symbols), LES with $D=132 \Delta$ (solid lines), and $D=82 \Delta$ (dashed lines). First and third column: $z=0.7 D$; second and fourth column: $z=3.6 D$. From bottom to top: $D_{e} / D=0.73 ; D_{e} / D=0.45 ; D_{e} / D=0.33$. 

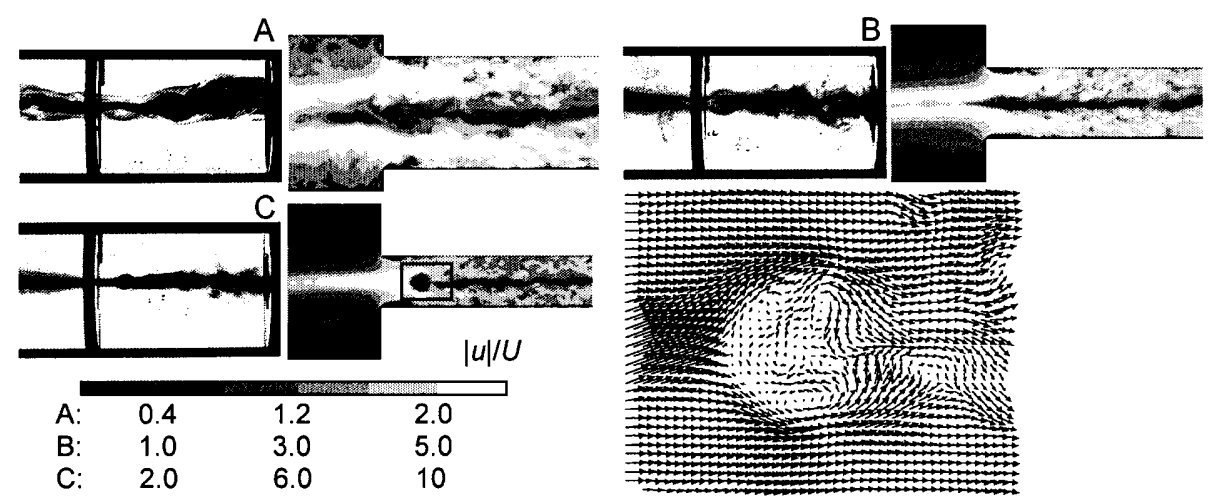

Figure 6. Visualization of the flow near the contraction. Experiment (dye injection) [15], and LES snapshots with $D=132 \Delta$ (the contours indicate absolute velocity). A: $D_{\mathrm{e}} / D=0.73$ (Reexp $\left.=200, R e_{L E S}=1,900\right) ; \mathrm{B}: D_{e} / D=0.45\left(R e_{\text {exp }}=650, \operatorname{Re}_{L E S}=1,900\right) ; \mathrm{C}: D_{e} / D=0.33\left(R e_{\text {exp }}=1,600\right.$, $\left.R e_{L E S}=1,900\right)$. The rectangle in part $\mathrm{C}$ relates to the velocity vector field.

ence/absence of "shoulders" in the profiles of axial velocity. Quantitatively, the spatial resolution is (as it was for agitated flow systems) a key issue. The simulations with $D_{e}=0.33 D$ that have the largest velocity gradients clearly lack spatial resolution. The trend, however, is in the right direction: the finer the grid, the better the predictions.

In the experiments, vortex breakdown was observed in the exit pipe. Vortex breakdown [17] is an abrupt change in the structure of a swirling flow. In bubble type vortex breakdown, a stagnation point is spontaneously formed at some position along the axis of the swirling flow. Vortex breakdown can trigger a laminar-turbulent transition. Figure 6 shows the experimental evidence: In cases B and C, a bubble-shaped dye structure can be observed just downstream the contraction indicating vortex breakdown. Upstream (left) of the bubble, a laminar vortex core can be observed, downstream (right) there clearly is turbulent flow. The simulations excellently represent this flow structure (a one-on-one comparison can in fact only be made for case $\mathrm{C}$, where the experimental and numerical Reynolds number are close); and the laminar-turbulent transition associated with it. The stagnation point character of the breakdown is clear from the vector plot in Fig. 6. We may conclude that the (isotropic) Smagorinsky SGS model is able to resolve the major features of this swirling flow system.

\section{Summary}

A procedure for large-eddy simulations in complexly shaped flow geometries has been presented. The three-dimensional, time-dependent flow information that can be generated this way might be beneficial in modeling industrial processes. The latticeBoltzmann method provided us with a means to reach high spatial, and temporal resolution. At the same time, we have limited ourselves to uniform, cubic grids. In many applications, especially if boundary layer flow is of critical importance, local grid refinement is required. In the lattice-Boltzmann community, various options for 
non-uniform, and even arbitrarily shaped grids have been proposed (e.g. [18]). To my best knowledge, no generally accepted method has evolved yet.

The assumptions underlying the Smagorinsky subgrid-scale model have been discussed. In the applications presented here, many of these assumptions could not be met: stirred tanks do not have equilibrium turbulence; the turbulence in swirling flows is highly anistropic. Still, the results presented on these flow systems show fairly good agreement with experimental data.

\section{References}

1. Lesieur, M., Métais, O.: New trends in large-eddy simulation of turbulence. Annual Review of Fluid Mechanics 28 (1996) 45

2. Rothman, D.H., Zaleski, S.: Lattice-gas cellular automata. Cambridge University Press, Cambridge (1997)

3. Chen, S., Doolen, G.D.: Lattice Boltzmann method for fluid flows. Annual Review of Fluid Mechanics 30 (1998) 329

4. Hou, S., Sterling, J., Chen, S., Doolen, G.D.: A lattice-Boltzmann subgrid model for high Reynolds number flows. Fields Institution Communications 6 (1996) 151

5. Somers, J.A.: Direct simulation of fluid flow with cellular automata and the latticeBoltzmann equation. Applied Scientific Research 51 (1993) 127

6. Eggels, J.G.M.: Direct and large-eddy simulations of turbulent fluid flow using the latticeBoltzmann scheme. International Journal of Heat and Fluid Flow 17 (1996) 307

7. Smagorinsky, J.: General circulation experiments with the primitive equations: 1 . The basic experiment. Monthly Weather Review 91 (1963) 99

8. Eggels, J.G.M.: Direct and large eddy simulation of turbulent flow in a cylindrical pipe geometry. PhD thesis, Delft University of Technology (1994)

9. Van Driest, E.R.: On turbulent flow near a wall. Journal of Aerodynamic Science 23 (1956) 1007

10. Germano, M., Piomelli, U., Moin, P., Cabot, W.: A dynamic subgrid-scale eddy-viscosity model. Physics of Fluids A 3 (1991) 1760

11. Schäfer, M., Yianneskis, M., Wächter, P., Durst, F.: Trailing vortices around a $45^{\circ}$ pitched-blade impeller. AIChE Journal 44 (1998) 1233

12. Derksen, J.J.,. Van den Akker, H.E.A.: Large eddy simulations on the flow driven by a Rushton turbine. AIChE Journal 45 (1999) 209

13. Escudier, M.P., Bornstein, J., Maxworthy, T.: The dynamics of confined vortices. Proceedings of the Royal Society London A 382 (1982) 335

14. Derksen, J.J., Van den Akker, H.E.A.: Simulation of vortex core precession in a reverseflow cyclone. AIChE Journal 46 (2000) 1317

15. Escudier, M.P., Bornstein, J., Zehnder, N.: Observations and LDA measurements of confined turbulent vortex flow. Journal of Fluid Mechanics 98 (1980) 49

16. Hoekstra, A.J.: Gas flow field and collection efficiency of cyclone separators, $\mathrm{PhD}$ thesis, Delft University of Technology (2000)

17. Escudier, M.P.: Vortex breakdown: observations and explanations. Progress in Aerospace Sciences 25 (1988) 189

18. He, X., Doolen, G.: Lattice-Boltzmann method on curvilinear coordinate system: flow around a circular cylinder. Journal of Computational Physics 134 (1997) 306 